\title{
OS CONCEITOS DA ARQUITETURA BIOCLIMÁTICA E SUA RELAÇÃO COM A EFICIÊNCIA ENERGÉTICA NAS EDIFICAÇÕES
}

\author{
Julie Cristina de Oliveira MEULAM ${ }^{1}$ \\ https://orcid.org/0000-0002-9959-8772 \\ Thayara TONIETTO ${ }^{2}$ \\ https://orcid.org/0000-0003-2992-1201
}

Reginaldo Ferreira SANTOS 3

https://orcid.org/0000-0002-7745-9173

Jair Antonio Cruz SIQUEIRA 4

https://orcid.org/0000-0002-8140-444X

\begin{abstract}
Resumo: o objetivo deste é avaliar quais as técnicas da arquitetura bioclimática são eficientes no consumo de energia elétrica e como auxiliam nos níveis de eficiência energética dos edifícios. $O$ conhecimento das corretas práticas arquitetônicas bioclimáticas são fundamentais na elaboração de projetos mais eficientes energeticamente. Dessa maneira foram abordadas as estratégias arquitetônicas bioclimáticas, como a orientação do edifício, a iluminação e ventilação natural, os brises soleil, espelhos d'agua e a utilização da vegetação. Pode-se entender que um edifício é energeticamente mais eficiente que outro por proporcionar as mesmas condições ambientais com menor consumo de energia. Os benefícios da eficiência energética não são apenas voltados para as medidas tradicionais de redução de demanda energia, também diminuem a poluição do ar local, geram uma economia nos orçamentos públicos, melhoram a saúde e a qualidade de vida da população, proporcionam segurança energética, minimizam os gases de efeito estuda, entre outros. Conclui-se com base nas informações analisadas, que as técnicas da arquitetura bioclimática aplicadas nas construções, ocasionaram efeitos na redução de temperatura nos ambientes em comparação as áreas que não empregavam tais métodos. Os resultados deste estudo demonstraram também que as técnicas arquitetônicas bioclimáticas influenciam parcialmente no microclima local onde estão sendo empregadas.
\end{abstract}

Palavras Chave: Bioclimatologia. Energia elétrica. Conforto térmico.

Abstract: The objective of this is to evaluate which techniques of bioclimatic architecture are efficient in the consumption of electric energy and how they help in the energy efficiency levels of the buildings. Knowledge of correct bioclimatic architectural practices is critical in designing more energy efficient designs. In this way the bioclimatic architectural strategies were approached, such as the orientation of the building, the natural lighting and ventilation, the brises soleil, water mirrors and the use of vegetation. It can be understood that one building is more energy efficient than another because it provides the same environmental conditions with lower energy consumption. The benefits of energy efficiency are not only directed at traditional energy demand reduction measures, they also reduce local air pollution, generate savings in public budgets, improve the health and quality of life of the population, provide energy security, minimize effect gases studies, among others. It is concluded based on the analyzed information, that the techniques of bioclimatic architecture applied in the buildings, caused effects in the temperature reduction in the environments in comparison to

${ }^{1}$ Mestranda em Engenharia de Energia na Agricultura, Cascavel, Paraná. juliemeulam.enf@hotmail.com. ${ }^{2}$ Mestranda em Engenharia de Energia na Agricultura, Cascavel, Paraná. leticia_tonietto@hotmail.com. ${ }^{3}$ Prof. Dr. da Universidade Estadual do Oeste do Paraná, Cascavel, Paraná. Reginaldo.santos@unioeste.br. ${ }_{4}^{4}$ Prof. Dr. da Universidade Estadual do Oeste do Paraná, Cascavel, Paraná. jair.siqueira@unioeste.br. 


\section{International Journal of Environmental Resilience Research and Sciente (IJERRS)}

Revista Internacional Resiliência Ambiental Pesquisa e Ciência Saciedade 5.๑ Resiliência Ambiental

the areas that did not use such methods. The results of this study also demonstrated that bioclimatic architectural techniques partially influence the local microclimate where they are being used.

Key Words: Bioclimatology. Electrical energy. Thermal comfort.

\section{INTRODUÇÃO}

A eficiência energética só entrou em pauta na agenda mundial nos anos de 1970 devido aos crescentes aumentos no preço do petróleo, momento em que se evidenciou que as reservas dos recursos fosseis teriam custos crescentes, tanto financeira quanto ambientalmente (EMPRESA DE PESQUISA ENERGÉTICA - EPE, 2010). Segundo a Agência Internacional de Energia (IEA, 2018) a eficiência energética é fundamental para garantir um sistema energético seguro, confiável, acessível e sustentável para o futuro. O manual "Indicadores de Eficiência Energética: Fundamentos e Estatísticas" (IEA, 2014a) aponta que eficiência energética é usar menos energia para fornecer o mesmo serviço.

Em uma abordagem sobre os benefícios da eficiência energética a IEA (2014b), revelou uma ampla gama de potenciais impactos positivos, tais como diminuição dos gases de efeito estufa, segurança energética, alivio da pobreza, melhora da saúde e bemestar da população, geração de empregos, diminuição da poluição do ar local, economia nos orçamentos públicos entre outros. O Conselho Mundial de energia (World Energy Council, 2008) verificou que, em todo o mundo, 76 países possuem políticas de eficiência energética, o que representa $83 \%$ do consumo mundial de energia, das regiões representativas a América do Norte e a Europa possuem $100 \%$ dos países com políticas energéticas, a América Latina $68 \%$, a Ásia $73 \%$, a África $54 \%$ e o Oriente Médio $44 \%$.

A IEA (2013) cita alguns exemplos de países e suas estratégias para melhorar o desempenho energético em seus países, entre eles destacam-se a Holanda com projetos de infraestrutura de energia a fim de garantir o interesse nacional, a Suécia que planeja que até 2030 toda sua frota veicular seja com combustíveis livre de fóssil

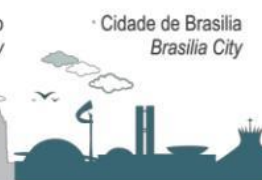




\section{International Journal of Environmental}

\section{Resilience Research and Science (IJERRS)}

Revista Internacional Resiliência Ambiental Pesquisa e Ciência Sociedade 5.0 Resiliência Ambiental

e implementação de política de taxação de dióxido de carbono, a Austrália que está ampliando a identificação e a adoção de oportunidades de eficiência energética e a Nova Zelândia que está elaborando um programa para aumentar o número de casas quentes e secas através do isolamento contribuindo na economia de energia.

No Brasil, o expressivo crescimento econômico que ocorreu no início do século $X X$, oriundo principalmente do processo de industrialização e o crescimento demográfico acompanhado de uma acelerada urbanização, se refletiu, principalmente, na demanda de energia primaria (EPE, 2007). Entretantosomente nos anos de 1980 é que surgiram, no Brasil, os primeiros programas com o intuito de promover a eficiência energética e o gerenciamento da demanda de energia, esses programas foram propostos no intuito de estimular o uso eficiente de energia elétrica, combater os desperdícios, bem como o consumo eficiente dos recursos naturais não renováveis (PEREIRA; WEISS, 2016; NASCIMENTO, 2015).

$\mathrm{Na}$ arquitetura um edifício é energeticamente mais eficiente que outro por proporcionar as mesmas condições ambientas com menor consumo de energia (LAMBERTS; DUTRA; PEREIRA, 2014). A arquitetura bioclimática interage com o clima local, com a edificação sempre centralizada no conforto ambiental do usuário e também quais repercussões ambientais causará ao planeta, evitando o desperdício dos recursos naturais, poupando energia, e contribuindo com a qualidade de vida do usuário (CORBELLA, YANNAS, 2009; CORBELLA, CORNER, 2011).

Com o exposto acima, o objetivo do trabalho consiste em abordar a arquitetura bioclimática e a eficiência energética, identificando técnicas e estratégias arquitetônicas para a bioclimatologia, tais como a orientação da edificação, a utilização de brises soleil, o uso de vegetação, a utilização da água, a ventilação e iluminação natural, entre outros e relacionar como a arquitetura bioclimática auxilia nos índices de eficiência energética nas construções.

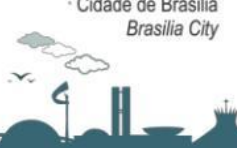




\section{International Journal of Environmental Resilience Research and Sciente (IJERRS)}

Revista Internacional Resiliência Ambiental Pesquisa e Ciência Saciedade 5.0 Resiliência Ambiental

\section{Construindo usando os princípios da arquitetura bioclimática}

Segundo Lamberts, Dutra e Pereira (2014) ao se construir uma edificação com conceitos bioclimáticos, alguns fatores devem ser analisados nas primeiras etapas do projeto, tais como as condições climáticas da região que incluem a temperatura do ar, a umidade, os ventos, a radiação solar, entre outros, deve-se analisar o terreno e a implantação da edificação, pois esse fator determinará quais estratégias bioclimáticas deverão serem incorporadas.

\section{Orientação da edificação}

A orientação correta das edificações é de suma importância e devia ser a principal exigência arquitetônica, estudos mostram que um edifício bem orientado pode reduzir em até $20 \%$ no consumo de energia da obra (ALWETAISHI et al., 2017). A orientação da edificação determina três fatores, as vistas, a luz solar e a ventilação natural (RUIZ e BANDERA, 2014). Sendo os dois últimos responsáveis pelas variações de temperaturas no interior dos ambientes e eles estão fortemente relacionados com a orientação da construção (SHUN, 2010).

Uma boa orientação deve levar em conta a trajetória solar e devem ser analisados na concepção projetual, pois controlam, diminuem ou aumentam o nível de incidência solar em determinando ambiente, parede ou afim (MONTERO, 2006). A orientação do edifício afeta o consumo de energia, devido a minimização gastos com refrigeração ou aquecimento mecânico e iluminação artificial, aumentando sua eficiência, também a orientação afeta a forma da edificação e o uso da transparência no mesmo (MARIN, 2017).

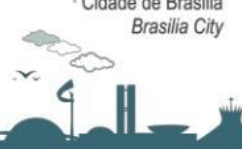




\section{International Juurnal of Environmental Resilience Research and Sciente (IJERRS)}

Revista Internacional Resiliência Ambiental Pesquisa e Ciência Saciedade 5.0 Resiliência Ambiental

\section{Ventilação natural e iluminação natural}

Para Frota e Schiffer (2005) a ventilação natural é responsável por renovar o ar no interior dos ambientes, melhorando então questões de salubridade e o conforto térmico. A ventilação natural nas edificações revela-se também como uma estratégia para diminuir impactos ambientais, pois reduz o uso dos sistemas de ar condicionado, diminuindo então o consumo de energia. O uso correto da ventilação natural, pode influenciar na economia de energia elétrica cerca de 31,9\%, de acordo com a localização e orientação da edificação, além do modelo da abertura escolhido (RUPP e GHISI, 2013).

A ventilação natural nos edifícios é realizada devido dois mecanismos; a ventilação por ação dos ventos, a força dos ventos causa o movimento do ar através do ambiente; e a ventilação por efeito chaminé (FROTA e SCHIFFER, 2005). Lamberts, Dutra e Pereira (2014) afirmam que a ventilação cruzada é umas das práticas mais eficazes nos ambientes e consiste na posição de duas aberturas em posições distintas.

Em relação a iluminação natural, existem diversos recursos arquitetônicos que podem ser utilizados afim de maximizá-la nos ambientes, como por exemplo, telhados com shed, prateleiras de luz, claraboias entre outras técnicas que facilitam a entrada de luz natural (LAMBERTS; DUTRA; PEREIRA, 2014). Entretanto a maneira mais comum e utilizada de se obter iluminação natural, é através da iluminação lateral, que corresponde as portas, janelas ou até mesmo as paredes envidraçadas (BARBOSA, 2010).

Didoné e Pereira (2009) observaram que o aproveitamento de luz natural na edificação propiciou uma redução no consumo de energia de $12 \%$ a $52 \%$, isto é, o aproveitamento da luz natural nos ambientes influenciou diretamente no consumo final de energia do edifício.

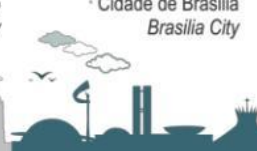




\section{International Journal of Environmental Resilience Research and Sciente (IJERRS)}

Revista Internacional Resiliência Ambiental Pesquisa e Ciência Saciedade 5.0 Resiliência Ambiental

\section{Brises}

Segundo Kamal (2013) o brise soleil ou quebra-sol, é um elemento de proteção solar simples, muito utilizado em regiões de clima quente, onde o excesso de calor interno, pode aumentar o consumo de energia para resfriar a edificação. De tal modo, de acordo com LIMA et al., (2011) sua função é impedir que os raios solares atinjam diretamente o interior dos ambientes, evitando ofuscamentos, contrastes e ajudando a combater o aquecimento interno.

Os brises são constituídos por lâminas, fixa ou moveis, no exterior da fachada e são organizados para interagir com a composição arquitetônica. Podem ser usados de diferentes formas nas edificações, como: horizontais, verticais e mistos (SCHERER, 2014). Também existem os brises móveis que podem ser regulados conforme a trajetória do sol (QUEIROZ e THIEME, 2016).

O sombreamentos dos edifícios tem sido a técnica mais sustentável e simples que existe para reduzir as cargas de resfriamento nas edificações, nas regiões de clima quente e tropical o usa-se o ar condicionado de 7 a 8 meses no ano, assim, os métodos de sombreamento melhoram a economia de energia de 10\% a 40\% (KAMAL, 2013).

\section{Espelhos d'agua}

A água é um elemento indispensável à vida, e se apresenta como importante parte integrante em projetos arquitetônicos e paisagísticos atuando como parte da climatização promovendo conforto aos ambientes próximos a ela (MASIEIRO, SOUZA, 2011). Os corpos d'agua funcionam como uma fonte natural de refrigeração, visto que a água possui um efeito positivo sobre o microclima das áreas circundantes devido ao resfriamento natural ocasionado a partir do processo de evaporação (WONG et. al, 2012).

Pesquisas pesquisa sobre o microclima criado por espelhos d'agua observaram 


\section{International Journal of Environmental}

\section{Resilience Research and Science (IJERRS)}

Revista Internacional Resiliência Ambiental Pesquisa e Ciência Sociedade 5.0 Resiliência Ambiental

que a diferença entre a umidade relativa do ar entre pontos próximos ao corpo d'agua e afastado e varia em média $10 \%$ a $12 \%$ e a que a temperatura máxima do ar atinge uma média de $4^{\circ} \mathrm{C}$ a $4,22^{\circ} \mathrm{C}$, ou seja, os locais próximos ao espelho d'agua possuem maior umidade e menor temperatura em ralação a locais mais afastados, ou seja, os locais próximos ao espelho d'agua possuem maior umidade e menor temperatura em ralação a locais mais afastados (VAVALLO, ROMERO, 2015; JIN, SHAO, ZHANG, 2017).

\section{Vegetação (telhado verde, painéis verdes, paisagismo)}

A vegetação na cidade deve ser realizada de forma que cumpra seu papel efetivo, proporcionando sombreamento quando necessário e amenizando os efeitos da radiação solar, visto que a vegetação atua como um filtro das radiações que são absorvidas pelo solo e pelas edificações, essa absorção das radiações promove o arrefecimento dos ambientes próximos (ROMERO, 2000). Quanto maior for o número de folhas de cobertura por unidade de terra maior será a capacidade de filtragem da vegetação, desta forma, a ordem de suficiência é a seguinte: arvores, arbustos e gramas (ROMERO, 2001).

Shinzato (2009) em sua pesquisa sobre os impactos da vegetação nos microclimas urbanos observou que a diferença média de temperatura do ar nas áreas arborizadas em relação as ruas circunvizinhas é de $1,5^{\circ} \mathrm{C}$, já as temperaturas das superfícies dos solos embaixo da copa mostraram diferença média de $23^{\circ} \mathrm{C}$, essa diferença é ocasionada pelo sombreamento das arvores de copas densas. O estudo ainda mostrou que o uso das arvores é um método eficaz para amenizar o efeito de ilhas de calor nas metrópoles, uma vez que minimiza o aquecimento de materiais como o asfalto e o concreto e a liberação da radiação de ondas longas acumuladas durante a noite, entretanto os efeitos da vegetação não vão muito além dos limites das áreas verdes, ficando restritos apenas ao local onde está inserida. Buyadi, Mohd e Misni (2015) também constataram que as temperaturas nas áreas com vegetação

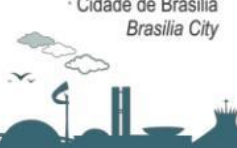




\section{International Journal of Environmental Resilience Research and Sciente (IJERRS)}

Revista Internacional Resiliência Ambiental Pesquisa e Ciência Saciedade 5.0 Resiliência Ambiental

foi $1,32^{\circ} \mathrm{C}$ menor do que na área construída (residencial), os autores também frisaram que, mesmo em áreas verdes menores com plantio linear ao longo de estradas ou parques vizinhos dentro da área residencial, há notáveis benefícios de resfriamento do microclima das áreas circundantes pelas sombras das arvores durante o processo de evapotranspiração durante o dia.

Em relação ao uso de vegetação no telhado verde Shafique, Kim e Raquif (2018) expressam a importância desta estratégia no intuito de tornar as cidades mais seguras, sustentáveis e resilientes as mudanças climáticas. Besir e Cuce (2018) em seu levantamento acerca de coberturas e fachadas verdes indicam que estas, são soluções fundamentais para a redução do consumo de energia relacionado com edifícios e as emissões de gases de efeito estufa, entre os benefícios encontrados na pesquisa podem ser destacados:

- A penetração de calor dos telhados do edifício no verão pode ser reduzida em cerca de $80 \%$;

- Os telhados verdes consomem entre $2,2 \%$ a $16,7 \%$ menos energia no verão do que os telhados convencionais;

- A demanda de aquecimento dos edifícios pode ser reduzida em $10 \%$ a $30 \%$ através de superfícies verdes;

- A diferença de temperatura entre os telhados convencionais e verdes no inverno é de cerca de $4^{\circ} \mathrm{C}$, enquanto que é de cerca de $12^{\circ} \mathrm{C}$ no verão;

- A diferença de temperatura entre a parede viva e a parede nua é de $1^{\circ} \mathrm{C}$ a $31,9^{\circ} \mathrm{C}$.

\section{METODOLOGIA}

Este artigo revisou a literatura global a partir de diversas fontes, isto é, revisões de livros, artigos de pesquisas e estudos de caso. Pesquisa com um número de palavras chaves diferentes que incluem energy efficiency, bioclimatic architecture, natural ventilation, natural lighting, brises, vegetation microclimate, Water Bodies,

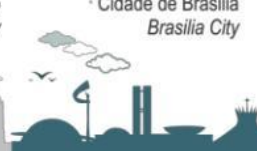




\section{International Journal of Environmental Resilience Research and Sciente (IJERRS)}

Revista Internacional Resiliência Ambiental Pesquisa e Ciência Saciedade 5.0 Resiliência Ambiental

vegetation urban microclimate, green roofs, Google scholar e diferentes revistas foram usadas para encontrar as informações úteis.

Esta revisão propicia uma visão geral sobre a arquitetura bioclimática, seus conceitos e princípios e quais são as estratégias arquitetônicas para se construir bioclimaticamente. Para salientar a importância do assunto foram apresentados estudos que demonstram os benefícios das estratégias, tanto a nível residencial quanto a nível urbano.

Trata-se de uma pesquisa bibliográfica, definida como descritiva, pois o objetivo é revelar as etapas de uma revisão sistemática de literatura, seus desafios e vantagens. O trabalho realizado é de caráter qualitativo, a análise qualitativa é realizada de forma dedutiva durante o levantamento dos dados do referencial teórico. No método de levantamento bibliográfico foram utilizados livros, artigos e periódicos nacionais e internacionais, no período de 2000 ano até 2018.

\section{DISCUSSÃO}

O excessivo consumo de energia dos edifícios aumentou drasticamente na última década em todo o mundo (EL-DARWISH; GOMAA, 2017), e ações políticas significativas foram desenvolvidas em diversos países no intuito de tornar mais eficiente e consciente o uso de energia. Esta revisão concentrou-se em estudos sobre a influência das técnicas de arquitetura bioclimática no melhor desempenho energético das edificações.

Uma série de estudos (GAITANI; MIHALAKAKOU; SANTAMOURIS; 2007; MANZANO-AGUGLIARO et al., 2015 E BECCALI et al., 2018) demonstrou que, os princípios da arquitetura bioclimática quando aplicados melhoram as condições de conforto térmico das edificações e do meio urbano, desta forma os projetos arquitetônicos devem ser aplicados na tentativa de tentar reduzir a demanda por energia o quanto possível em relação ao clima do local da construção.

Esta revisão decidiu coletar evidências atuais da influência da arquitetura

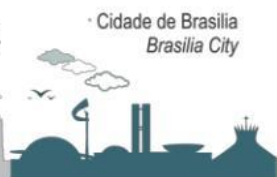




\section{International Journal of Environmental Resilience Research and Sciente (IJERRS)}

Revista Internacional Resiliência Ambiental Pesquisa e Ciência Saciedade 5.0 Resiliência Ambiental

bioclimática na diminuição do estresse térmico residencial e urbano. Todos os trabalhos analisados apresentaram como resultado de suas pesquisas uma diminuição da temperatura dos ambientes onde foram implementadas as técnicas bioclimáticas quando comparadas as áreas que não utilizavam essas técnicas.

Este estudo propõe que o uso de práticas arquitetônicas bioclimáticas melhoram a eficiência energética das edificações, entretanto os resultados deste estudo evidenciam que as técnicas arquitetônicas bioclimáticas influenciam parcialmente no microclima local onde estão sendo empregadas.

\section{CONSIDERAÇÕES FINAIS}

Observou-se que todas as técnicas bioclimáticas apresentadas, quando aplicadas, surtiram efeitos positivos na redução da temperatura dos ambientes por elas circundados.

Em relação aos corpos d'agua estas atenuam a temperatura apenas nas regiões mais próximas ao local onde estão situadas. A água esfria o ambiente ao longo do dia, entretanto a noite, devido a uma alta da umidade do ar, a atmosfera retém mais calor fazendo com que assim a temperatura neste período fique um pouco alta, o que torna esse sistema o menos eficaz no consumo de energia elétrica.

A orientação do edificou também se mostrou menos eficaz, visto que depende de outros fatores como localização, orientação do edifício e modelo de aberturas para que atinja o máximo de sua eficácia.

Os brises e a ventilação e iluminação natural são técnicas eficazes no consumo de energia elétrica, principalmente em países tropicais, visto que sua maior eficiência se apresenta apenas em determinadas épocas do ano.

Já a vegetação na cidade se apresentou muito eficaz na redução da temperatura do ambiente, principalmente na redução de ilhas de calor, porém sua eficácia não atinge áreas mais longes de onde está inserida a vegetação. Em contrapartida, os

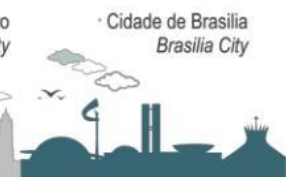




\section{International Journal of Environmental}

\section{Resilience Research and Sciente (IJERRS)}

Revista Internacional Resiliência Ambiental Pesquisa e Ciência Sociedade 5.0 Resiliência Ambiental

telhados verdes se mostraram a técnica mais eficiente na redução da temperatura interna dos ambientes bem como na economia de energia dos edifícios, por proporcionarem resfriamento dos ambientes no verão e aquecimento no inverno.

As pesquisas nesta área, devem considerar todos os fatores ambientais e suas variáveis, além do papel do processo de construção da arquitetura bioclimática. $\mathrm{O}$ conforto térmico dos usuários dos ambientes é de suma importância, porém priorizar as políticas de redução de consumo de energia e eficiência energética nos edifícios é um fator relevante para que a arquitetura bioclimática atinja seus objetivos.

\section{REFERÊNCIAS}

ALWETAISHI, M. et al. Investigation of school building microclimate using advanced energy equipment: Case study. Environmental Engineering Research, República da Coréia, v. 23, n. 1, p. 10-20, jul. 2017. Disponível em: <http://eeer.org/upload/eer-23-1-10.pdf>. Acesso em: 15 mai. 2018.

BARBOSA, C. V. T. Percepção da iluminação no espaço da arquitetura: preferências humanas de iluminação em ambientes de trabalho. 2010. 238p. Tese (Doutorado em Arquitetura e Urbanismo) Faculdade de Arquitetura e Urbanismo da Universidade de São Paulo, São Paulo, 2010.

BECCALI, M. et al. Vernacular and bioclimatic architecture and indoor thermal comfort implications in hot-humid climates: An overview. Science Direct. V. 82, p. 1726 - 1736, February 2018.

BESIR, A. B.; CUCE, E. Green roofs and facades: A comprehensive review. Renewable and Sustainable Energy Reviews, v. 82, part 1, p. 915 - 939, 2018.

BUYADI, S. N. A.; MOHD, W. M. N. W..; MISNI, A. Vegetation's Role on Modifying Microclimate of Urban Resident. Procedia - Social and Behavioral Sciences. [S.L], v. 202, p. 400 - 407, 2015.

CORBELLA, O. D.; YANNAS, S. Em busca de uma arquitetura sustentável para os trópicos. Rio de Janeiro: 2 Edição. Editora Revan, 2009. p.308

CORBELLA, O.; CORNER, V. N. Manual de arquitetura bioclimática tropical. Para a redução de consumo energético. Rio de Janeiro: 1 Edição. Editora Revan, 2011. p.111

DIDONÉ, E.L.; PEREIRA, F.O.R. O potencial da luz natural na economia de energia elétrica para a iluminação artificial. Revista Tecnológica, edição especial ENTECA, p. 24-34, 2009.

EL-DARWISH, I.; GOMAA, M. Retrofitting strategy for building envelopes to achieve energy efficiency. Alexandria Engineering Journal. V. 56, p. 579 - 589, December 2017.

EPE - EMPRESA DE PESQUISA ENERGÉTICA. Nota técnica DEA 14/10: Avaliação da Eficiência energética na indústria e nas residências no horizonte decenal (2010-2019). Ministério de Minas e Energia. Serie: Estudos da Demanda. Rio de Janeiro, 2010. Disponível em: <http://www.epe.gov.br/sitespt/publicacoes-dados-abertos/publicacoes/PublicacoesArquivos/publicacao-245/topico-

270/20100809_4[1].pdf> Acesso em: 10/05/2018. 


\section{International Journal of Environmental}

\section{Resilience Research and Sciente (IJERRS)}

Revista Internacional Resiliência Ambiental Pesquisa e Ciência Saciedade 5.0 Resiliência Ambiental

EPE - EMPRESA DE PESQUISA ENERGÉTICA. PLANO NACIONAL DE ENERGIA 2030. Ministério de Minas e Energia: Colaboração Empresa de Pesquisa Energética, Brasília, 2007. Disponível em: < http://www.mme.gov.br/documents/10584/1139260/Plano+Nacional+de+Energia+2030+\%28PDF\%29/ ba957ba9-2439-4b28-ade5-60cf94612092?version=1.1 > Acesso em: 12/05/2018.

FROTA, A. B; SCHIFFER, S. R. Manual de conforto Térmico. São Paulo: $8^{a}$ edição. Editora Studio Nobel, 2005, p.248

GAITANI, N.; MIHALAKAKOU, G.; SANTAMOURIS, M. On the use of bioclimatic architecture principles in order to improve thermal comfort conditions in outdoor spaces. Science Direct. V. 42, p. 317 - 324, Janeiro 2007.

IEA - INTERNATIONAL ENERGY AGENCY. Energy Efficiency Indicators: Fundamentals on Statistics, 2014a. Disponível em: <

https://www.iea.org/publications/freepublications/publication/IEA_EnergyEfficiencyIndicatorsFundame ntalsonStatistics.pdf> Acesso em: 17/05/2018.

IEA - INTERNATIONAL ENERGY AGENCY. Energy Efficiency. 2018. Disponível em: $<$ https://www.iea.org/topics/energyefficiency/> acesso em: 10/05/2018.

IEA - INTERNATIONAL ENERGY AGENCY. Energy Policy: Highlights. 2013.

IEA - INTERNATIONAL ENERGY AGENCY. Taking a multiple benefits approach to energy efficiency. In: Capturing the Multiple Benefits of Energy Efficiency, 2014b. Disponível em:

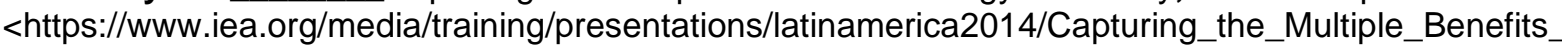
of_Energy_Efficiency_2014_Executive_Summary.pdf> acesso em: 10/05/2018

JIN, Hong; SHAO, Teng; ZHANG, Renlong. Effect of water body forms on microclimate of residential district. Energy Procedia, [S.L], v. 134, p. 256 - 265, 2017. Disponível em: < https://www.sciencedirect.com/science/article/pii/S1876610217347495> Acesso em 18/05/2018.

KAMAL, M. A. Le corbusier's solar shading strategy for tropical environment: a sustainable approach. Jars (journal of architectural/planning research and studies), Tailândia, v. 10, n. 1, p. 19-26, jun. 2013. Disponível em: <http://www.tds.tu.ac.th/jars/download/jars/v101/02\%20muhammad.pdf> Acesso em: 12 mai. 2018

LAMBERTS, R.; DUTRA, L.; PEREIRA, F. O. R.; Eficiência energética na arquitetura. São Paulo: 3o Edição. Editora: Eletrobrás/Procel, 2014. p. 284

LIMA, K.M; et al. A influência de protetores solares na iluminação natural no edifício sede da Compesa. In.: ENCAC - Encontro Nacional de Conforto no Ambiente Construído e ELACAC Encontro Latino Americano de Conforto no Ambiente Construído, Búzios, 2011

MANZANO-AGUGLIARO, F. et al. Review of bioclimatic architecture strategies for achieving thermal comfort. Science Direct. V. 49, p. 763 - 755, September 2015.

MARIN, D. M. The impact of building orientation on energy usage. Using simulation software IDA ICE 4.7.1. 2017. 33p. Tese (Mestrado em Engenharia de Energia) - University of Gävle. Faculty of Engineering and Sustainable Development. Suécia, 2017

MASIERO, É.; SOUZA, L. C. L. de. INFLUÊNCIA DE UM CORPO D'ÁGUA SOBRE O MICROCLIMA URBANO EM SÃO JOSÉ DO RIO PRETO - SP. XI Encontro Nacional de conforto no ambiente contruído e VII Encontro Latino americano de conforto no ambiente construído, Buzios/RJ, 2011. Disponível em: <http://www.infohab.org.br/encac/files/2011/topico2artigo5.pdf>. Acesso em: 18/05/2018.

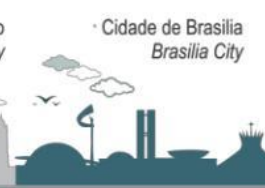




\section{International Journal of Environmental}

\section{Resilience Research and Sciente (IJERRS)}

Revista Internacional Resiliência Ambiental Pesquisa e Ciência Sociedade 5.0 Resiliência Ambiental

MONTERO, J. I. P. Ventilação e iluminação naturais na obra de João Filgueiras Lima "Lelé": Estudo dos hospitais da rede Sarah Kubitschek Fortaleza e Rio de Janeiro. 2006. 262p. Tese (Mestrado em Arquitetura e Urbanismo) - Escola de Engenharia da Universidade de São Carlos, São Carlos 2006

NASCIMENTO, R. L. Política De Eficiência Energética No Brasil. [S.L.]: Câmara dos Deputados, 2015. $31 \mathrm{p}$.

PEREIRA, A.; WEISS, M. O Setor Elétrico e as Novas Políticas de Eficiência Energética. Caderno Opinião FGV ENERGIA, 2016. Disponível em:

<https://bibliotecadigital.fgv.br/dspace/bitstream/handle/10438/19246/Coluna\%20Opiniao\%20Novemb ro\%20Amaro\%20e\%20Mariana.pdf> Acesso em: 11/05/2018.

QUEIROZ, J.; THIEME, C. Edifício comercial: uso de proteção solar na avaliação da iluminação e refrigeração através de simulação. In: ENCONTRO NACIONAL DE TECNOLOGIA DO AMBIENTE CONSTRUÍDO, 2016. São Paulo. Anais. Porto Alegre: ANTAC, 12p.

ROMERO, M. A. B. A Arquitetura Bioclimática do Espaço Público. Brasília: Editora Universidade de Brasília, 2001.

ROMERO, M. A. B. Princípios Bioclimáticos para o desenho Urbano. $2^{\circ}$ ed. São Paulo:

ProEditores, 2000.

RUIZ, R; BANDERA, F. Importance of orientation in building energy-savings. World sbk 2014 barcelona, Espanha, p. 327-334, out. 2014. Disponível em:

<http://www.irbnet.de/daten/iconda/cib_dc28305.pdf> Acesso em: 12 mai.2018

RUPP, R. F.; GHISI, E. Potencial de economia de energia elétrica em edificações comerciais híbridas localizadas em Florianópolis, SC. ANTAC, Porto Alegre, v. 13, n. 1, p. 143-160, jan./mar. 2013. Disponível em: <http://www.seer.ufrgs.br/index.php/ambienteconstruido/article/view/23611/24738> Acesso em: 15.mai.2018

SCHERER, M. J. Cortinas verdes na arquitetura: desempenho no controle solar e na eficiência energética de edificações. 2014. 187p.Tese (Doutorado em Arquitetura) - Universidade Federal do Rio Grande do Sul. Porto Alegre, 2014.

SHAFIQUE, M.; KIM, R.; RAFIQ, M. Green roof benefits, opportunities and challenges - A review. Renewable and Sustainable Energy Reviews, v. 90, p. 757 - 773, 2018.

SHINZATO, P. Os Impactos da Vegetação nos Microclimas Urbanos. 2009. 173 p. Dissertação de Mestrado - FAUUSP. São Paulo, 2009.

SHUN, L. T. Effects of building orientation on the residential property price: an empirical study in telford garden. 2010. 84p. Tese (Licenciatura em ciências) - The University of Hong Kong. China, 2010. Disponível em: <http://hub.hku.hk/handle/10722/130982> Acesso em: 16.mai.2018

VAVALO, H. M.; ROMERO, M. B. O microclima criado por espelhos d'água: estudo de caso do espelho d'água do congresso nacional. Paranoá Cadernos De Arquitetura E Urbanismo, Brasilia, n. 14, p. 09-17, jan. 2015. Disponível em:

<http://periodicos.unb.br/index.php/paranoa/article/view/16927/12028>

WONG et. al. Influence of Water bodies on Outdoor Air Temperature in Hot and Humid Climate. Paper presented at the Reston, VA: ASCE copyright Proceedings of the 2011 International Conference on Sustainable Design and Construction.

WORLD ENERGY COUNCIL. Energy Efficiency Policies around the World: Review and Evaluation. London, 122 p. 2008. 